\title{
Editorial: New Challenges in Marine Pollution Monitoring
}

\author{
Juan Bellas ${ }^{1 *}$, Ketil Hylland ${ }^{2}$ and Thierry Burgeot ${ }^{3}$ \\ ${ }^{1}$ Centro Oceanográfico de Vigo, Instituto Español de Oceanografía, IEO, Vigo, Spain, ${ }^{2}$ Department of Biosciences, University \\ of Oslo, Oslo, Norway, ${ }^{3}$ Unit of Biogeochemistry and Ecotoxicology, Institut Français de Recherche pour l'Exploitation de la \\ Mer, Nantes, France
}

Keywords: marine pollution, ecotoxicology, biomonitoring, water quality, bioaccumulation

\author{
Editorial on the Research Topic
}

\author{
New Challenges in Marine Pollution Monitoring
}

\section{INTRODUCTION}

There is abundant evidence that anthropogenic activities have polluted all compartments of the oceans, from the poles to the tropics, by different physical, chemical, and biological stressors. Chemical pollution is particularly tackled here with focus on legacy pollutants and newly emerging man-made compounds (xenobiotics) or anthropogenic forcing in the increase of natural chemical substances. It has been estimated that more than 100,000 chemicals are currently on the market [ECHA (European Chemicals Agency), 2017], and thousands of new substances are being introduced every year due to industrialization, intensive agriculture, and urban development. This has led to a continuous flow of chemical products to the oceans that have the potential to alter the structure of ecosystems by causing changes in the biotic communities that constitute them.

Traditionally, the assessment of marine chemical pollution would exclusively be based on chemical analysis of a limited set of potential pollutants in selected environmental matrices, and a comparison between their levels with those found in pristine areas not being subjected to direct human pressures. However, such chemical assessment of pollution only offers a partially suitable approach to the question of how marine organisms and ecosystem functioning are affected by pollutants. This can only be answered by means of an integrated assessment including both chemical analyses and biological tools that quantitatively link the levels of pollutants with their ecological effects, including new contaminants for which no analytical techniques have yet been developed. Ideally, we aim to detect disturbances caused by pollutants before ecosystems are affected. So we need sensitive indications for pollution effects that provide an early warning to allow taking measures to avoid ecological damage. As for this concern a unique effort based on a European consensus, has been developed by ICES/OSPAR (Davies and Vethaak, 2012).

A new challenge in marine pollution monitoring is also based on the harmonization of two European Union directives for the protection of the marine environment, the Water Framework Directive (WFD, 2000/60/CE) and the Marine Strategy Framework Directive (MSFD, 2008/56/CE). The latter established a legislative context demanding the use of effect-based tools for the assessment of pollution. These two directives were constructed according to two different strategies to assess the status of continental and coastal water ecosystems, following either a risk assessment approach (WFD) or an ecosystem approach (MSFD).

This Research Topic comprises 14 studies including original research articles, method developments, reviews, perspectives, and technology reports covering several aspects of marine pollution monitoring: integrated methodologies, distribution, and levels of legacy and emerging 
pollutants and their impact on populations and communities of marine organisms, as well as new techniques for marine pollution monitoring.

\section{INTEGRATED ASSESSMENT OF POLLUTION}

An integrated approach for the assessment of marine pollution generates large and heterogeneous datasets including variables with very different metrics and distributions. All of these must be considered when selecting the method for data analysis. In this Research Topic, Regoli et al. applied a Weight of Evidence (WOE) model to results from the monitoring of offshore platforms, based on multiple lines of evidence including chemical analyses and biological responses. The assessment of environmental quality was summarized in terms of hazard quotients, providing a useful tool for the application of risk assessment in environmental management. Lehtonen et al. assessed pollution impacts in coastal areas of Finland with a WOE approach, using caged mussels. The integration of chemical and biological effect data revealed that the health of marine organisms in the area of study is being altered by pollution. Parmentier et al. assessed the effects of tributyltin (TBT) on the brown shrimp (Crangon crangon) and suggest that long-term endocrine disruption effects was the reason for previously unexplained declines in $C$. crangon and other crustacean populations in the German Bight. Förlin et al. studied perch (Perca fluviatilis) populations at reference sites from the Swedish National Monitoring Program where a decline in fish health had been previously observed. Alterations in several biological responses, confirmed by mRNA expression levels, were related to higher concentrations of natural, bioactive brominated compounds measured in the perch, attributed to algal blooms in the Baltic Sea. Yang et al. studied the distribution and levels of persistent organic pollutants in surface sediments of Qingduizi Bay. Polychlorinated biphenyls (PCBs) and hexachlorocyclohexane ( $\mathrm{HCH})$ levels were attributed to their extensive historical use in adjacent areas. However, the DDT profile showed recent usage and fresh inputs from aquaculture activities, with levels that could be potentially toxic to marine organisms.

\section{POLLUTANTS OF EMERGING CONCERN}

Pollutants of emerging concern are substances that have recently raised awareness due to their widespread use and presence in the environment and might represent a threat to ecosystems or to human health. These chemicals are not adequately regulated and urgent information is needed about their environmental distribution, bioaccumulation potential, and ecotoxicity. Among the substances of emerging concern, little attention has been paid to metals. Romero-Freire et al. reviewed the concentrations of Less-Studied Technology-Critical Elements (LSTCEs) (Nb, $\mathrm{Ta}, \mathrm{Ga}, \mathrm{In}, \mathrm{Ge}, \mathrm{Te}$ ) in marine water and biota. Limited information of LSTCEs is available for estuarine and coastal waters and further efforts are needed to develop appropriate analytical procedures and certified reference materials. Among the pollutants of emerging concern, there has recently been increasing interest in microplastics (MPs). A number of studies have demonstrated that MPs pollution is widespread and ubiquitous in the marine environment, with potential to cause harm to biota. Tramoy et al. assessed plastic inputs from the Seine River basin to the sea using two methods, a statistical modeling approach and field results from floating booms. Both methods yielded similar estimates of plastic fluxes to the sea, ranging from 1,100 to $5,900 \mathrm{t} / \mathrm{yr}$. Assumptions and uncertainties of both approaches were characterized. Schönlau et al. studied the levels of aryl hydrocarbon receptor (AhR) active compounds on field-deployed MPs. Bioassay-derived TCDD (2,3,7,8-tetrachlorodibenzo-p-dioxin) equivalents were calculated with the DR CALUX ${ }^{\circledR}$ assay to evaluate the potential toxicity of hydrophobic organic compounds (HOCs) sorbed to MPs. The bioassay identified two bioactive fractions in the polymers and proved to be a valuable complement to chemical analysis in risk assessment of MP-sorbed compounds. Cormier et al. investigated the toxicity of organic pollutants sorbed to MPs using the zebrafish embryo test. No embryotoxicity was detected for virgin or spiked MPs, but oxybenzone- and benzo[a]pyrenespiked MPs induced EROD activity. These results indicate that the standard acute embryo test would not be sufficiently sensitive to detect effects of MPs, and protocols including sublethal endpoints would be needed.

\section{NEW TECHNIQUES AND TECHNOLOGY}

The availability of suitable techniques and methods has typically hindered the study of environmental pollutants and their impact on marine ecosystems. The development of new techniques is crucial to overcome analytical limitations and other methodological difficulties to advance in the knowledge of their environmental behavior and effects. Fauvelle et al. developed an analytical method based on a single solid-phase extraction procedure followed by gas and liquid chromatography coupled with high resolution mass spectrometry (GC-MS and LC-HRMS) for the quantification of 40 organic compounds in natural seawater. The procedure, which allowed the quantification of five classes of legacy and emerging contaminants, was validated and applied to real seawater samples. Tato and Beiras compared the sensitivity of Tisochrysis lutea (T-iso), formerly Isochrysis galbana, with other microalgal species recommended for regulatory toxicity testing, using the 72-h growth-rate inhibition test. In general, T-iso showed higher sensitivity for most groups of toxicants than other species, meeting the acceptability criteria, therefore its use in marine toxicity testing is recommended. Paredes and Bellas present a perspective on the application of cryopreservation and cryobanking techniques to marine pollution monitoring. These techniques offer a potential solution to ensure the availability of biological material of stable quality throughout the year. A background of existing knowledge is presented and further development is discussed, including the need for extensive comparative testing with both fresh and cryopreserved biological material. Cyr et al. present an ocean glider-compatible fluorescence sensor capable 
of continuously measuring polycyclic aromatic hydrocarbons (PAHs) concentrations in real time. The performance of the sensor was tested in laboratory with oil products and in three different deployments: an experimental flume tank, an urban harbor, and an off-shore oil and gas installation. Paris et al. studied the use of subsea dispersant injection (SSDI) in response to the Deepwater Horizon oil spill. Extensive BP Gulf Science Data were used to quantify petroleum dynamics throughout the spill, revealing no significant effect of the SSDI on the oil vertical distribution and concentration. These results question an uncritical SSDI application for deep-sea blowouts.

\section{CONCLUSIONS}

Marine pollution monitoring is a fundamental component in current environmental legislation which aims to preserve and protect marine ecosystems more effectively, and promoting their sustainable use. The articles of this Research Topic present some of the most important challenges to advance in this field. Integrated approach schemes need to improve data

\section{REFERENCES}

Davies, I. M., and Vethaak, A. D. (2012). Integrated Marine Environmental Monitoring of Chemicals and their Effects. ICES Cooperative Research Report No. 315, 277.

ECHA (European Chemicals Agency) (2017). Guidance for Identification and Naming of Substances Under REACH and CLP. Version 2.1. ECHA-16-B-37.1EN, Helsinki, 127.

Conflict of Interest: The authors declare that the research was conducted in the absence of any commercial or financial relationships that could be construed as a potential conflict of interest. comparability between biological responses, which is frequently not satisfactorily done due to both confounding factors and methodological variability. A crucial knowledge gap is also the elaboration of internationally agreed assessment criteria for both environmental pollutants and biological responses. The knowledge about the environmental behavior and ecotoxicity of pollutants of emerging concern, including microplastics, is particularly relevant for integrative monitoring purposes, as well as the development of new approaches and technologies in marine pollution monitoring.

\section{AUTHOR CONTRIBUTIONS}

All authors listed have made a substantial, direct and intellectual contribution to the work, and approved it for publication.

\section{ACKNOWLEDGMENTS}

We thank all authors, reviewers, and editors that have contributed to this Research Topic.
Copyright (c) 2020 Bellas, Hylland and Burgeot. This is an open-access article distributed under the terms of the Creative Commons Attribution License (CC BY). The use, distribution or reproduction in other forums is permitted, provided the original author(s) and the copyright owner(s) are credited and that the original publication in this journal is cited, in accordance with accepted academic practice. No use, distribution or reproduction is permitted which does not comply with these terms. 